\title{
Schottky Barrier Parameters of Pd/Ti Contacts on N-Type InP Revealed from I-V-T And C-V-T Measurements
}

\author{
D. Subba Reddy ${ }^{1}$, Matli Bhaskar Reddy ${ }^{2}$, N. Nanda Kumar Reddy ${ }^{1}$, Varra Rajagopal Reddy ${ }^{\text {* }}$ \\ ${ }^{1}$ Department of Physics, Sri Venkateswara University, Tirupati, India \\ ${ }^{2}$ Government Degree College, Puttur, India \\ E-mail: reddy_vrg@rediffmail.com
}

Received January 5, 2011; revised February 26, 2011; accepted February 27, 2011

\begin{abstract}
We report on the current-voltage $(\mathrm{I}-\mathrm{V})$ and capacitance-voltage $(\mathrm{C}-\mathrm{V})$ characteristics of the $\mathrm{Pd} / \mathrm{Ti} / \mathrm{n}-\mathrm{InP}$ Schottky barrier diodes (SBDs) in the temperature range $160-400 \mathrm{~K}$ in steps of $40 \mathrm{~K}$. The barrier heights and ideality factors of Schottky contact are found in the range $0.35 \mathrm{eV}(\mathrm{I}-\mathrm{V}), 0.73 \mathrm{eV}(\mathrm{C}-\mathrm{V})$ at $160 \mathrm{~K}$ and 0.63 $\mathrm{eV}(\mathrm{I}-\mathrm{V}), 0.61 \mathrm{eV}(\mathrm{C}-\mathrm{V})$ at $400 \mathrm{~K}$, respectively. It is observed that the zero-bias barrier height $\Phi_{b o}$ decreases and ideality factor $\mathrm{n}$ increase with a decrease in temperature, this behaviour is attributed to barrier inhomogeneities by assuming Gaussian distribution at the interface. The calculated value of series resistance $\left(\mathrm{R}_{\mathrm{s}}\right)$ from the forward I-V characteristics is decreased with an increase in temperature. The homogeneous barrier height value of approximately $0.71 \mathrm{eV}$ for the $\mathrm{Pd} / \mathrm{Ti}$ Schottky diode has been obtained from the linear relationship between the temperature-dependent experimentally effective barrier heights and ideality factors. The zero-bias barrier height ( $\Phi_{b o}$ ) versus $1 / 2 \mathrm{kT}$ plot has been drawn to obtain evidence of a Gaussian distribution of the barrier heights and values of $\bar{\Phi}_{b 0}=0.80 \mathrm{eV}$ and $\sigma_{0}=114 \mathrm{mV}$ for the mean barrier height and standard deviation have been obtained from the plot, respectively. The modified Richardson $\ln \left(\mathrm{I}_{0} / \mathrm{T}^{2}\right)-$ $\left(q^{2} \sigma_{0}^{2} / 2 k^{2} T^{2}\right)$ versus $1000 / \mathrm{T}$ plot has a good linearity over the investigated temperature range and gives the mean barrier height $\left(\bar{\Phi}_{b 0}\right)$ and Richardson constant $\left(\mathrm{A}^{*}\right)$ values as $0.796 \mathrm{eV}$ and $6.16 \mathrm{Acm}^{-2} \mathrm{~K}^{-2}$ respectively. The discrepancy between Schottky barrier heights obtained from I-V and C-V measurements is also interpreted.
\end{abstract}

Keywords: Schottky Barrier Parameters, I-V-T and C-V-T Measurements, Pd/Ti Schottky Contacts, N-Type InP, Gaussian Distribution

\section{Introduction}

Metal-semiconductor (MS) structures are important research tools in the characterization of new semiconductor materials, and at the same time, the fabrication of these structures plays a vital role in constructing some useful devices in technology [1-3]. Indium phosphide (InP) is a promising III-V compound semiconductor material for high-speed electrical and optoelectronic devices. Due to its direct bandgap, high electron mobility, high saturation velocity and breakdown voltage which are very important in electronic devices [4-5]. But, a serious limitation of InP Schottky barrier diodes is the low barrier height and large leakage currents. However, low barrier height Schottky diodes of InP seem to be a good candidate for the application of zero-bias Schottky detector diodes [5].
Most studies of Schottky barrier diodes (SBDs) formed on n-InP were limited to the determination of the Schottky barrier height (SBH) at room temperature (RT) by measuring either the current-voltage (I-V) characteristics or the capacitance-voltage $(\mathrm{C}-\mathrm{V})$ characteristics of the diodes [6-8]. Therefore, analysis of the current-voltage (I-V) characteristics of the SBDs at room temperature only does not give detailed information about their conduction process or the nature of the barrier formed at the metal-semiconductor (M-S) interface. On the other hand, the temperature dependent studies of the Schottky contacts enable one to understand different aspects of conduction mechanisms [9-11]. The observed current-voltage (I-V) characteristics of the real SBDs usually deviate from the ideal thermionic emission (TE) model. The strong dependence of both barrier height and the ideality factor on tempera- 
ture, the difference in BHs obtained from different methods and the non-linearity of the Richardson's plot are the factors associated with the deviation from the TE model $[9,12,13]$. Explanation of the possible origin of such anomalies has been proposed, taking into account the interface state density distribution [11], quantum-mechanical tunneling [14-16], image force lowering [14] and most recently the lateral distribution of barrier height inhomogeneities $[17,18]$. Another way to describe the inhomogeneity is to assume a Gaussian distribution of the barrier heights over the contact area [19].

Schottky barrier diodes (SBDs) formed by depositing various metals on $n$ - type InP have been studied over a wide temperature range [20-25]. Cetin et al. [20] studied the temperature dependent electrical characteristics of $\mathrm{Au} / \mathrm{n}$-InP SBDs in the temperature of $80-320 \mathrm{~K}$. They showed that barrier heights and ideality factors varied in the range of $0.274-0.516 \mathrm{eV}$ and $2.32-1.05$, respectively in the measured temperature range. Bhaskar Reddy et al. [21] investigated the current-voltage-temperature (I-V-T) characteristics of $\mathrm{Pd} / \mathrm{Au} / \mathrm{InP}$ SBDs in the temperature range of $220-400 \mathrm{~K}$. They reported that the barrier heights, ideality factors and series resistance were strongly temperature dependent. Soylu et al. [22] investigated the current-voltage (I-V) and capacitance-voltage (C-V) characteristics of the gold Schottky contacts on moderately doped $\mathrm{n}$-InP in the temperature range of $60-300 \mathrm{~K}$. They found that the ideality factor $\mathrm{n}$ of the diode decreases while the corresponding zero-bias SBH increasing with an increase in the temperature. Ashok kumar et al. [23] evaluated the Schottky barrier parameters of $\mathrm{Pd} / \mathrm{Pt} / \mathrm{n}$-InP Schottky barrier diode in the temperature range of $230-410 \mathrm{~K}$. They found that the increase in ideality factor and decrease in barrier height with a decrease in temperature and explained such behaviour on the basis of the thermionic emission with Gaussian distribution of the barrier heights at the interface. Cimilli et al. [24] investigated the temperature dependent electrical characteristics of Ag Schottky contacts on n-InP in the temperature range of $30-320 \mathrm{~K}$. They reported that the decrease in the experimental barrier height calculated from I-V measurement and an increase in the ideality factor with a decrease in the temperature which was due to the barrier inhomogeneities at the metal-semiconductor interface. More recently, Naik et al. [25] investigated the temperature dependent currentvoltage $(\mathrm{I}-\mathrm{V})$ and capacitance-voltage $(\mathrm{C}-\mathrm{V})$ characteristics of the $\mathrm{Au} / \mathrm{Ni} / \mathrm{n}$-InP SBDs in the temperature range of $210-420 \mathrm{~K}$. They showed that the barrier parameters varied significantly with temperature.

The main aim of the present study is to fabricate $\mathrm{Pd} / \mathrm{Ti}$ Schottky contacts to n-type InP and measured the current-voltage $(\mathrm{I}-\mathrm{V})$ and capacitance-voltage $(\mathrm{C}-\mathrm{V})$ char- acteristics in the temperature range of $160-400 \mathrm{~K}$ by steps of $40 \mathrm{~K}$. In this work, titanium (Ti) is selected as a first contact layer because it has low work function and it provides the lowest forward voltage drop as well. The palladium $(\mathrm{Pd})$ is used as over layer on $\mathrm{Ti}$ contact because it reacts with $\mathrm{InP}$ at low temperatures and improved contact morphology. The resultant temperaturedependent barrier characteristics of the $\mathrm{Pd} / \mathrm{Ti} / \mathrm{n}$-InP Schottky contacts have been interpreted on the basis of the existence of Gaussian distribution of the barrier height around a mean value due to barrier height inhomogeneities prevailing at the metal-semiconductor (M-S) interface.

\section{Experimental Procedure}

Liquid Encapsulated Czokralski (LEC) grown undoped n-InP (111) samples with carrier concentration of $4.5 \times$ $10^{15} \mathrm{~cm}^{-3}$ are used in the present work. Prior to metal deposition, the InP wafer is degreased for $5 \mathrm{~min}$ in warm organic solvents like trichloroethylene, acetone and methanol sequentially by means of ultrasonic agitation to remove contaminants followed by rinsing in deionized (DI) water and then the samples are dried in high purity $\mathrm{N}_{2}$ gas. The cleaning procedure is followed by a $60 \mathrm{~s}$ dip in $\mathrm{HF}(49 \%)$ and $\mathrm{H}_{2} \mathrm{O}(1: 10)$ to remove the native oxides from the front surface of the wafer. After this etching process the wafer is immediately loaded into the deposition chamber of e-beam evaporation system. For ohmic contact, indium (In) is evaporated on to the non-polished side of the wafer with a thickness of $500 \AA$. Then the ohmic contacts are formed by thermal annealing at $350{ }^{\circ} \mathrm{C}$ for $60 \mathrm{~s}$ in $\mathrm{N}_{2}$ atmosphere. Finally, $\operatorname{Ti}(200 \AA) / \operatorname{Pd}(300 \AA)$ metals are deposited on the polished side of the InP wafer as circular dots with a diameter of $0.7 \mathrm{~mm}$ as Schottky contacts by electron beam evaporation system. All evaporation processes are carried out in a vacuum coating unit at about $7 \times 10^{-6}$ mbar. Metal layer thickness as well as deposition rates are monitored with the help of a digital quartz crystal thickness monitor. The deposition rates are about $1.0 \AA_{\mathrm{s}}^{-1}$. The current-voltage (I-V) and capacitance-voltage $(\mathrm{C}-\mathrm{V})$ measurements are carried out by Keithley source measure unit (Model No.2400) and automated deep level spectrometer (SEMILAB DLS $83 \mathrm{D}$ ) in the temperature range $160-400 \mathrm{~K}$ by step of 40 $\mathrm{K}$ in the dark using temperature controller DLS 83D1 cryostat with a sensitivity of $\pm 1 \mathrm{~K}$.

\section{Results and Discussion}

\subsection{Analysis of Current-Voltage-Temperature (I-V-T) Characteristics}

Based on the thermionic emission theory, the current- 
voltage characteristics are given by the relation [1]

$$
I=I_{0} \exp \left(\frac{q V}{n k T}\right)\left[1-\exp \left(\frac{-q V}{k T}\right)\right]
$$

where $V$ is the applied voltage drop across the junction barrier, $q$ is the electronic charge, $k$ is the Boltzmann's constant, $T$ is the absolute temperature in Kelvin, $n$ is the diode ideality factor and $I_{0}$ is the saturation current and is expressed $[1,2]$ as

$$
I_{o}=A A^{*} T^{2} \exp \left(\frac{-q \Phi_{b 0}}{k T}\right)
$$

where $A$ is the diode area, $A^{*}$ is the effective Richardson's constant $\left(9.4 \mathrm{~A} \cdot \mathrm{cm}^{-2} \cdot \mathrm{K}^{-2}\right)$ based on effective mass $\left(\mathrm{m}^{*}=0.078 \mathrm{~m}_{\mathrm{o}}\right)$ of $\mathrm{n}$-InP [2] and $\Phi_{b o}$ is the apparent barrier height. The values of the barrier height $\left(\Phi_{b o}\right)$, and ideality factor (n) for the device are determined from the $y$ intercepts and slopes of the forward bias $\ln I$ versus $V$ plot at each temperature, respectively. The barrier height ( $\Phi_{b o}$ ) can be obtained by rewriting Equation (2) as

$$
\Phi_{b o}=\frac{k T}{q} \ln \left(\frac{A A^{*} T^{2}}{I_{0}}\right)
$$

The ideality factor ' $n$ ' is determined from the slope of the linear region of the plot of natural $\log$ of forward current versus forward bias voltage and is given by

$$
n=\frac{q}{k T}\left(\frac{\mathrm{d} V}{\mathrm{~d}(\ln I)}\right)
$$

The semi-logarithmic reverse and forward bias current-voltage characteristics of $\mathrm{Pd} / \mathrm{Ti} / \mathrm{n}$-InP Schottky barrier diode (SBDs) in the temperature range of 160-400 K in steps of $40 \mathrm{~K}$ are shown in Figure 1. It is observed that the leakage current increase with the increase in temperature is in the range $2.44 \times 10^{-7} \mathrm{~A}$ (at $160 \mathrm{~K}$ ) to $1.21 \times$

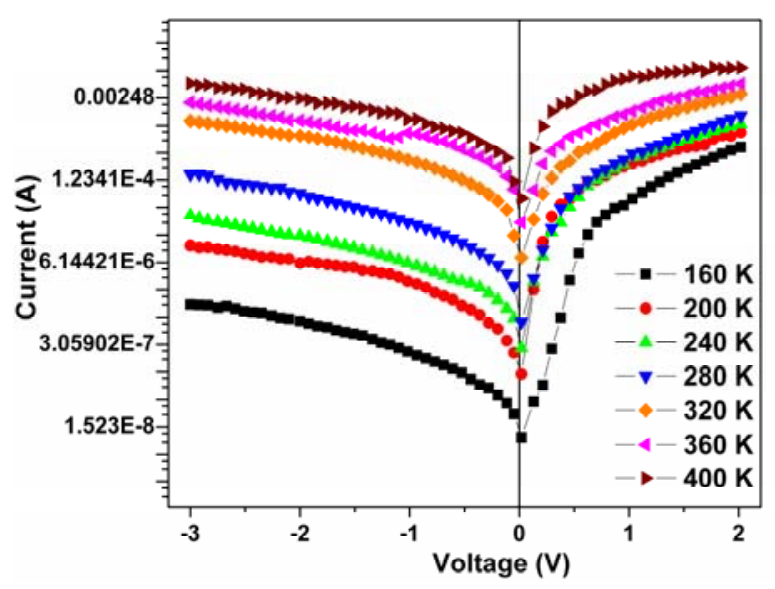

Figure 1. Semi-logarithmic reverse and forward bias current-voltage characteristics of $\mathrm{Pd} / \mathrm{Ti} / \mathrm{n}$-InP SBD in the temperature range of $160-400 \mathrm{~K}$.
$10^{-3} \mathrm{~A}($ at $400 \mathrm{~K}$ ) at $-1 \mathrm{~V}$. Measurements showed that the zero bias barrier height and the ideality factor (calculated using Equations (3) and (4)) of Pd/Ti/n-InP SBDs with temperature are $0.35 \mathrm{eV}$ and 3.75 at $160 \mathrm{~K}$ to $0.63 \mathrm{eV}$ and 1.73 at $400 \mathrm{~K}$ respectively. It is noted that the barrier height $(\mathrm{BH})$ is increased linearly from $0.35 \mathrm{eV}$ to 0.63 $\mathrm{eV}$ with the increase in temperature from $160 \mathrm{~K}$ to $400 \mathrm{~K}$, accompanied by a significant improvement of the ideality factor $\mathrm{n}$ from 3.75 to 1.73 . Interestingly it is noted that the experimental value of $\Phi_{b o}$ is found to increases with increase in temperature as shown in Figure 2. Since current transport across the metal-semiconductor interface is controlled by temperature, electrons at low temperature pass over the lower barriers and therefore current will flow through patches of the lower SBH and results in a larger ideality factor. In other words, as the temperature increases, more and more electrons have sufficient energy to overcome the higher barrier $[11,26]$. Figure 3 shows that the experimental values of $n$ (represented by open circles) increased with a decrease in temperature. The higher values of the ideality factor $(n>$ 1) indicate that there is a deviation from TE theory for current mechanism. Idealities greater than one can be attributed to the presence of a thick interfacial insulator layer between the metal and semiconductor $[1,27]$.

As shown in the Figure 1, the forward bias I-V characteristics are linear on a semi-logarithmic scale at low forward bias voltages but deviate considerably from linearity due to the effect of series resistance $\left(R_{S}\right)$. Temperature dependence of series resistance effect on the I-V characteristics of the $\mathrm{Pd} / \mathrm{Ti} / \mathrm{n}$-InP SBDs are investigated

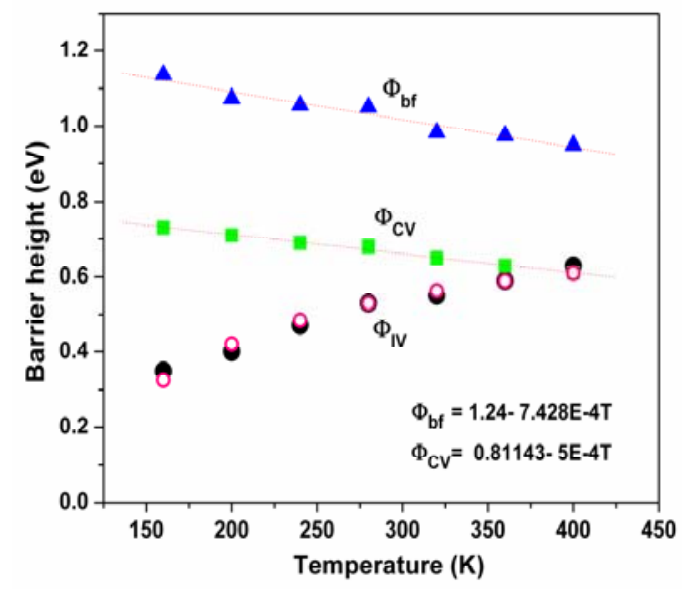

Figure 2. Temperature dependence of the zero-bias apparent barrier height, barrier height from $\mathrm{C}-\mathrm{V}$ data and flat band barrier height for Pd/Ti/n-InP SBD. The filled circles represent experimentally calculated barrier heights. The open circles represent estimated values of $\Phi_{a p}$ using Equation (13) with $\bar{\Phi}_{b 0} \quad(\mathbf{T}=\mathbf{0} \mathrm{K})=\mathbf{0 . 8 0 0 6} \mathrm{eV}$ and $\sigma_{0}=\mathbf{0 . 1 1 4 4}$ $\mathrm{V}$ values. 


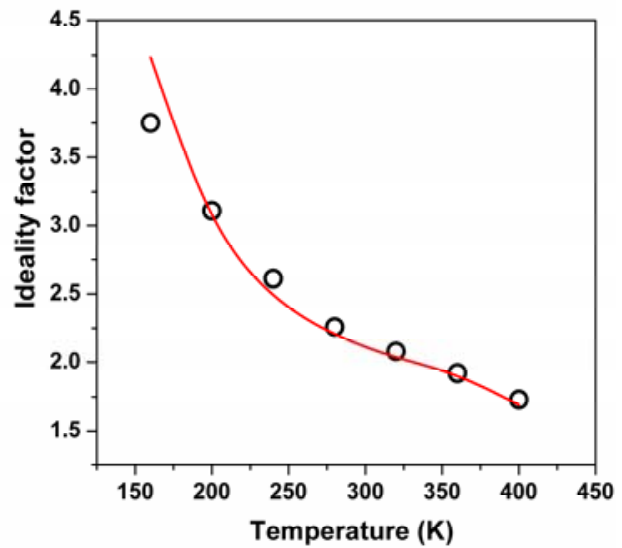

Figure 3. Temperature dependence of the ideality factor for $\mathrm{Pd} / \mathrm{Ti} / \mathrm{n}$-InP SBD in the range of 160 - $400 \mathrm{~K}$. The open circles shows the experimental ideality factors and continuous curve shows the estimated value of $\boldsymbol{n}_{\text {ap }}$ using Equation (14) with $\rho_{2}=\mathbf{0 . 2 5 0 4} \mathrm{V}$ and $\rho_{3}=-0.01417 \mathrm{~V}$.

in the temperature range of $160-400 \mathrm{~K}$. The resistance of the SBD is the sum of the total resistance value of the resistors in series and resistance in semiconductor device in the direction of current flow. The values of series resistance $\left(R_{s}\right)$ are achieved from the forward bias I-V data using the method developed by Cheung [28]. The forward bias current-voltage characteristics due to thermionic emission of a Schottky contact with the series resistance can be expressed as [1,29]

$$
\frac{\mathrm{d} V}{\mathrm{~d}(\ln I)}=I R_{S}+n\left(\frac{k T}{q}\right)
$$

Figure 4 shows the plot of $\mathrm{d} V / \mathrm{d}(\ln I)$ versus $I$ as a function of temperature. Equation (5) should give straight line for the data of downward curvature region in the forward bias I-V characteristics. Thus the slope of the plot of $\mathrm{d} V / \mathrm{d}(\ln I)$ versus $I$ gives $R_{S}$ as the slope and $\mathrm{n}(\mathrm{kT} / \mathrm{q})$ as the $\mathrm{y}$-axis intercept. The series resistance $\left(R_{S}\right)$ obtained for each temperature using Equation (5) and corresponding values are $1836 \Omega$ at $160 \mathrm{~K}$ and $272 \Omega$ at $400 \mathrm{~K}$. It is observed that the series resistance increases with decrease of temperature as shown in Figure 5. As can be seen in Figure 5, the decrease of $R_{S}$ with the increasing of temperature is believed to be due to factors responsible for increase in ideality factor $\mathrm{n}$ and lack of free carrier concentration at low temperatures [30]. The calculated zero-bias BH, ideality factors and series resistance of the Pd/Ti Schottky contacts as a function of temperature is given in the Table $\mathbf{1}$.

For the evaluation of the $\mathrm{BH}$, one may also make use of the Richardson plot of the saturation current. Equation (2) can be written as

$$
\ln \left(\frac{I_{0}}{T^{2}}\right)=\ln \left(A A^{*}\right)-\frac{q \Phi_{b 0}}{k T}
$$

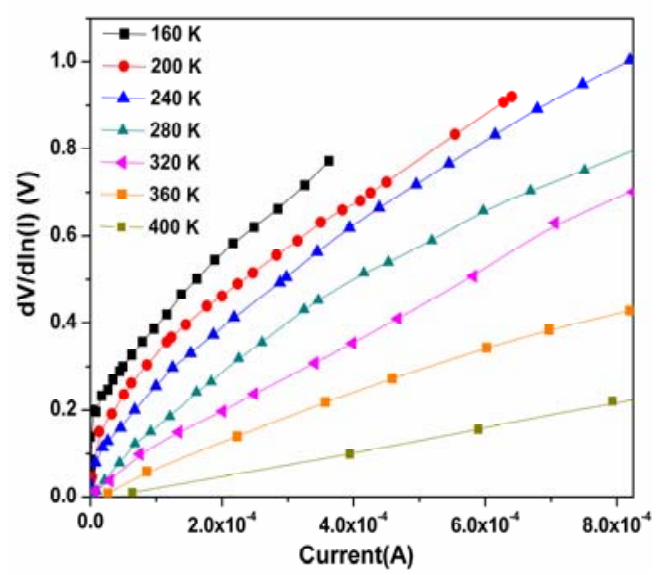

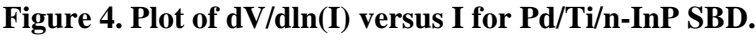

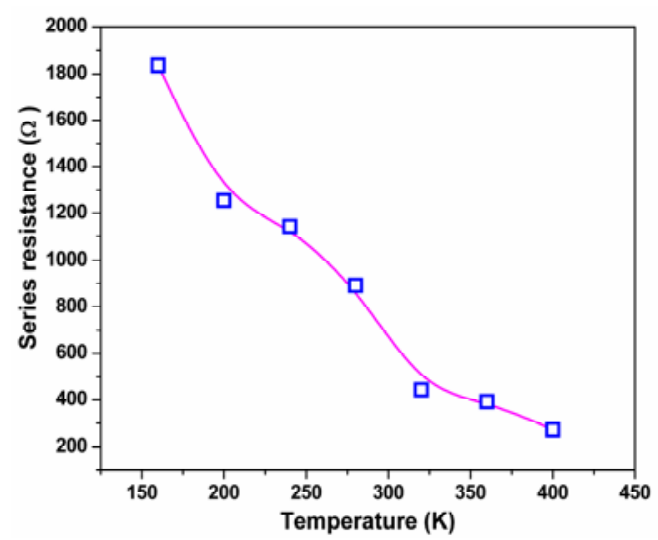

Figure 5. Temperature dependence of series resistance of $\mathrm{Pd} / \mathrm{Ti} / \mathrm{n}$-InP SBD in the temperature range of 160 - $400 \mathrm{~K}$.

The plot of $\ln \left(\mathrm{I}_{0} / \mathrm{T}^{2}\right)$ versus $10^{3} / \mathrm{T}$ is found to be nonlinear in the measured temperature range as shown in Figure 6. The non-linearity of the conventional $\ln \left(\mathrm{I}_{0} / \mathrm{T}^{2}\right)$ versus $10^{3} / \mathrm{T}$ is caused by the temperature dependence of the BH and ideality factor. The experimental data are seen to fit asymptotically to a straight line at higher temperatures only. According to Equation (6), the plot of $\ln \left(\mathrm{I}_{0} / \mathrm{T}^{2}\right)$ versus $10^{3} / \mathrm{T}$ yields a straight line with the slope giving the $\mathrm{BH}$ at $\mathrm{T}=0 \mathrm{~K}$ and the intercept giving the Richardson constant. An activation energy value of 0.27 $\mathrm{eV}$ from the slope of this straight line is obtained for the $\mathrm{Pd} / \mathrm{Ti}$ Schottky contact. The values of $\mathrm{A}^{*}$ obtained from the intercept of the straight line portion at the ordinate of the experimental $\ln \left(\mathrm{I}_{0} / \mathrm{T}^{2}\right)$ versus 1000/T plot in Figure 6 is equal to $2 \times 10^{-3} \mathrm{~A} \cdot \mathrm{cm}^{-2} \cdot \mathrm{K}^{-2}$, which is lower than the known value of $9.4 \mathrm{~A} \cdot \mathrm{cm}^{-2} \cdot \mathrm{K}^{-2}$. The deviation in the Richardson plot may be due to the effects of the imageforce, the effect of tunneling current through the potential barrier, the effect of recombination in the space charge region appearing at low voltage and the variation of the charge distribution near the interface [31]. Ac- 
Table 1. Ideality factors, series resistances and Schottky barrier heights of Pd/Ti Schottky contact on n-type InP in the temperature range of $160-400 \mathrm{~K}$.

\begin{tabular}{|c|c|c|c|c|c|}
\hline \multirow{2}{*}{$\begin{array}{c}\text { Temperature } \\
\text { (K) }\end{array}$} & \multirow{2}{*}{$\begin{array}{c}\text { Ideality } \\
\text { factor }(n)\end{array}$} & \multirow{2}{*}{$\begin{array}{c}\text { Series } \\
\text { resistance (Rs) } \\
(\Omega)\end{array}$} & \multicolumn{3}{|c|}{ Barrier heights } \\
\hline & & & $(\mathrm{eV}) \Phi_{b 0}$ & $(\mathrm{eV}) \Phi_{C-V}$ & $(\mathrm{eV}) \Phi_{b f}$ \\
\hline 160 & 3.75 & 1836 & 0.35 & 0.73 & 1.136 \\
\hline 200 & 3.11 & 1255 & 0.40 & 0.71 & 1.074 \\
\hline 240 & 2.61 & 1143 & 0.47 & 0.69 & 1.056 \\
\hline 280 & 2.26 & 893 & 0.53 & 0.68 & 1.051 \\
\hline 320 & 2.08 & 442 & 0.55 & 0.65 & 0.983 \\
\hline 360 & 1.92 & 392 & 0.59 & 0.63 & 0.975 \\
\hline 400 & 1.73 & 272 & 0.63 & 0.61 & 0.949 \\
\hline
\end{tabular}

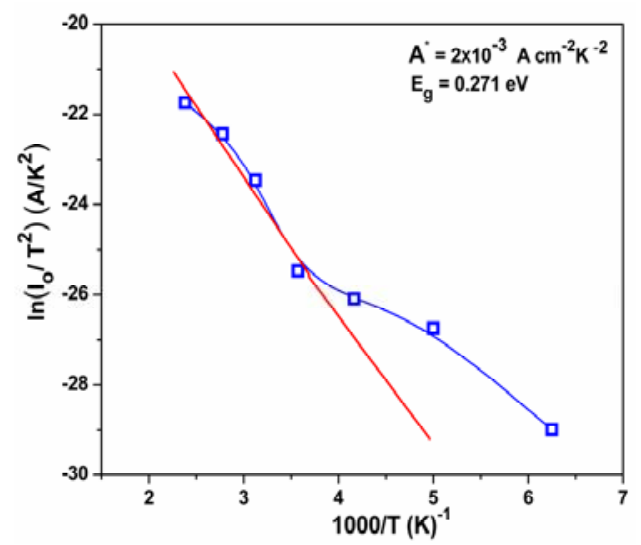

Figure 6. Richardson plot of $\ln \left(\mathrm{I}_{0} / \mathrm{T}^{2}\right)$ versus $10^{3} / \mathrm{T}$ for Pd/Ti/n-InP SBD.

cording to Horvath [32], the $A^{*}$ value obtained from the temperature dependence of the I-V characteristics may be affected by the lateral inhomogeneity of the barrier, and the fact that it is different from the theoretical value may be connected to a value of the real effective mass that is different from the calculated one.

It is observed that ideality factor $n$ is greater than unity which indicates that TE is not the only operative mechanism for current flow and is usually attributed to a SBH which is bias dependent. If the current transport is controlled by the thermionic field emission (TFE) theory, the relation between current and voltage can be expressed as $[33,34]$

$$
\begin{gathered}
I=I_{s} \exp \left[\frac{V}{E_{0}}\right] \\
\text { with } E_{0}=E_{00} \operatorname{coth}\left[\frac{q E_{00}}{k T}\right]=\frac{n_{t u n} k T}{q}
\end{gathered}
$$

where $E_{\mathrm{oo}}$ is the characteristic energy, which is related to the transmission probability of the carrier through the barrier given by

$$
E_{00}=\frac{h}{4 \pi}\left(\frac{N_{d}}{m_{e}^{*} \varepsilon_{s}}\right)^{\frac{1}{2}}
$$

where $h$ is the Planck constant $\left(h=6.626 \times 10^{-34} \mathrm{~J} \cdot \mathrm{sec}\right), N_{d}$ is the donor concentration, $\varepsilon_{s}$ is the semiconductor dielectric constant and $m_{e}^{*}$ is the electron effective mass.

In the case of our $\mathrm{Pd} / \mathrm{Ti} / \mathrm{n}$-InP SBDs with $N_{d}=2.63 \times$ $10^{15} \mathrm{~cm}^{-3}$ (from $\mathrm{C}-\mathrm{V}$ method at room temperature), $m_{e}^{*}=$ $0.077 m_{0}$ and $\varepsilon_{s}=12.4 \varepsilon_{0}$ the value of $E_{00}$ is found to be about $0.9738 \mathrm{meV}$. When considering the bias coefficient of the barrier height, $\beta=\partial \Phi_{b} / \partial \mathrm{V}$, Equation (8) can be written as $[32,35]$

$$
n_{\text {tun }}=\frac{E_{0}}{k T(1-\beta)}
$$

The theoretical temperature dependence of ideality factor for the case when the current through Schottky junction is dominated by the TFE is shown in Figure 7. The solid lines in Figure 7 are obtained by fitting Equation (8) to the experimental temperature dependence values of the ideality factor presented for different values of the characteristic energy $E_{\mathrm{oo}}$, without considering the bias coefficient of the $\mathrm{BH}, \beta=0$, for the $\mathrm{Pd} / \mathrm{Ti} / \mathrm{n}$-InP SBDs. The filled circles in the Figure 7 shows the temperature dependence values of ideality factor obtained from the experimental current-voltage (I-V) characteristics. From the Figure 7, it is observed that the experimental temperature dependence of ideality factor is in agreement with the curve (C) obtained with $E_{\mathrm{oo}}=15$ $\mathrm{meV}$ for the $\mathrm{Pd} / \mathrm{Ti} / \mathrm{n}$-InP SBD studied in the temperature range of $160-400 \mathrm{~K}$. Hence, there is a significant consistency between the theoretical and experimental characteristics as shown in Figure 7 (curve C).

The characteristic energy $E_{\mathrm{oo}}$ value is much larger than the theoretical value $0.973 \mathrm{meV}$ calculated for $n-I n P$. To understand the possible origin of the high characteristic energy values $E_{\mathrm{oo}}$, it should be underlined that $E_{\mathrm{oo}}$ is connected with the transmission probability [36,37]. The characteristic energy has been related to several effects such as the electric field present on the surface of the semiconductor [38], the existence of relatively a thick interfacial insulating layer between the deposited metal and semiconductor and the density of states. Therefore, any mechanism which enhances the electric field or the 


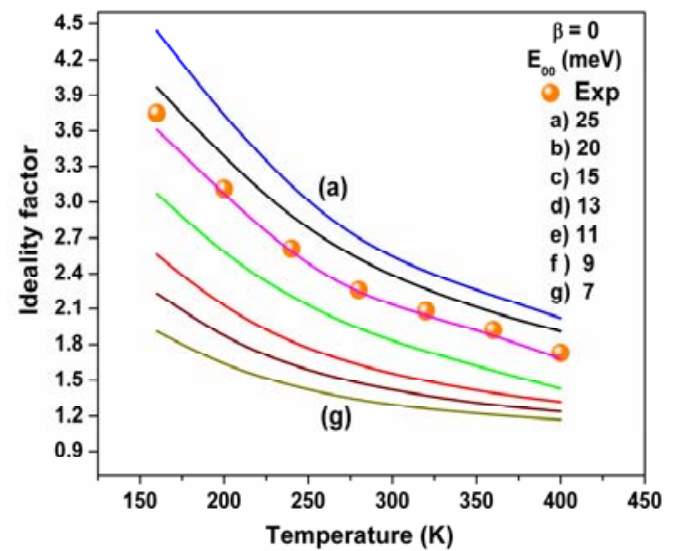

Figure 7. Theoretical temperature dependence of ideality factor for the case when the current through the junctions is dominated by the TFE with characteristic energy values $E_{00}$ according to Equation (8) (Solid lines) for Pd/Ti/n-InP SBD. The filled circles show the experimental temperature dependence values of the ideality factor obtained from I-V characteristics as shown in Figure 1.

density of states at the semiconductor surface will increase the TFE, and so the apparent $E_{\mathrm{oo}}[32]$.

Another way to correlate the obtained parameters ideality factor $n$ and barrier height $\Phi_{b o}$ is to calculate the flat-band $\mathrm{BH} \Phi_{b f}$. The $\mathrm{BH}$ decreases with decreasing temperature which is obtained from Equation (3) is called apparent or zero-bias BH. The BH obtained under flat-band condition is called the flat-band $\mathrm{BH}$ and is considered as the real essential quantity. In contrast to the case of the zero-bias $\mathrm{BH}$, the electric field in the semiconductor is zero under the flat-band condition and thus the semiconductor bands are flat, which eliminates the effect of tunneling and image force lowering that would affect the I-V characteristics and removes the influence of lateral inhomogeneity $[12,20]$. The flat-band barrier height $\left(\Phi_{b f}\right)$ is given by $[39,40]$

$$
\Phi_{b f}=n \Phi_{b 0}-(n-1)\left(\frac{k T}{q}\right) \ln \left(\frac{N_{c}}{N_{d}}\right)
$$

where $N_{c}$ is the effective density of states in the conduction band and $N_{d}$ the carrier concentration. The temperature dependent $N_{c}$ and $N_{d}$ values are used in calculating $\Phi_{C-V}$ and $\Phi_{b f}$. Figure 2 shows the variation of flatband barrier height $\Phi_{b f}$ as a function of the temperature. However, it is observed that $\Phi_{b f}$ increase with decreasing temperature. Similar phenomenon is also reported by the others $[20,22]$. The temperature dependence of the flat-band barrier height can be expressed as

$$
\Phi_{b f}(T)=\Phi_{b f}(T=0)+\alpha T
$$

where $\Phi_{b f}(T=0)$ and $\alpha$ are the flat-band barrier height extrapolated to the absolute zero and the temperature coefficient of the flat-band barrier height, respectively. The fit of Equation (12) to the experimental data (filled triangles) in Figure 2 yields $\Phi_{b f}(T=0)=1.24$ $\mathrm{eV}$ and $\alpha=-7.4285 \times 10^{-4} \mathrm{eVK}^{-1}$.

The ideality factor is simply a manifestation of the barrier uniformity and it increases for an inhomogeneous barrier [41]. A significant increase in the ideality factor and decrease in the SBH at low temperatures are possibly originated by inhomogeneities of thickness, and composition of the layer, non-uniformity of the interfacial charges or the presence of a thin insulating layer between the metal and the semiconductor $[9,10,26,39]$. Schmitsdorf [42] used Tung's theoretical approach and they found a linear correlation between the experimental zerobias SBHs and ideality factors. Figure 8 shows a plot of experimental $\mathrm{BHs}$ versus ideality factor with temperature for the $\mathrm{Pd} / \mathrm{Ti} / \mathrm{n}$-InP Schottky diode. The solid line in the Figure 8 is the least-squares fit to the experimental data. As can be seen from the Figure 8, there is a linear relationship between the experimental effective $\mathrm{SBHs}$ and the ideality factors of the Schottky contact. The extrapolation of the experimental BHs versus ideality factor plot to $n=1$ has given a homogeneous $\mathrm{BH}\left(\Phi_{\text {hom }}\right.$ ) of approximately $0.71 \mathrm{eV}$. The other $\mathrm{BH}$ values deviate from this value due to local inhomogeneities. A homogeneous $\mathrm{BH}$ of approximately $0.71 \mathrm{eV}$ obtained from the extrapolation of the least-square linear fitting to data to $\mathrm{n}=$ 1 (Figure 8) is in agreement with the value obtained by Ashok et al. [23] for the $\mathrm{Pd} / \mathrm{Pt} / \mathrm{n}-\mathrm{InP}$ Schottky barrier diodes.

The decrease in the $\mathrm{BH}$ with a decrease in temperature can also be explained by the lateral distribution of $\mathrm{BH}$ if the $\mathrm{BH}$ has a Gaussian distribution of the $\mathrm{BH}$ values over the Schottky contact area with the mean BH $\left(\bar{\Phi}_{b 0}\right)$ and standard deviation $\left(\sigma_{0}\right)$. The standard deviation is a measure of the barrier homogeneity. The Gaussian distribution of the BHs yields the following expression for

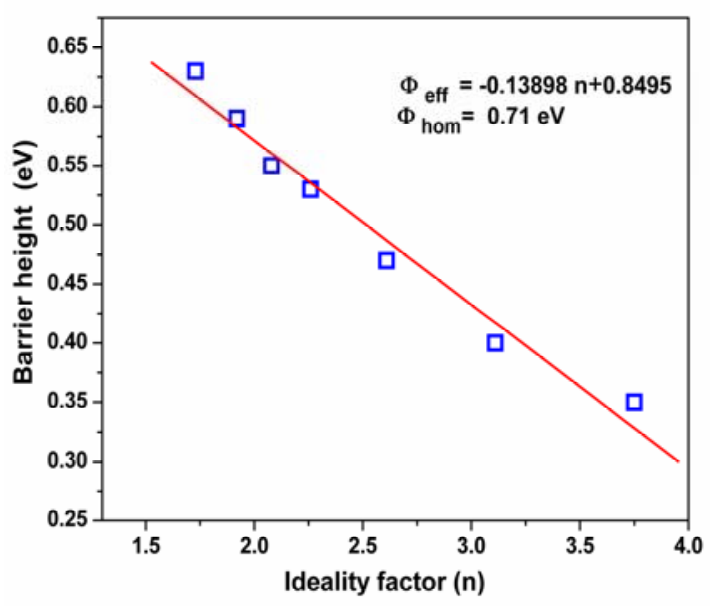

Figure 8. The zero-bias barrier height versus ideality factor for the Pd/Ti/n-InP SBD at different temperatures. 
the $\mathrm{BH}[9,10,12,20,22]$

$$
\Phi_{a p}=\bar{\Phi}_{b 0}(T=0)-\frac{q \sigma_{0}^{2}}{2 k T}
$$

where $\Phi_{a p}$ is the apparent $\mathrm{BH}$ measured experimentally. The same expression (Equation (13)) is used already by Song et al. [9] and also by Werner and Guttler [10] for the apparent $\mathrm{BH}$ construction. Usually the temperature dependence of $\sigma_{0}$ is small and it can be neglected. The observed variation of ideality factor with temperature in the model is given by [10]

$$
\left(\frac{1}{n_{a p}}-1\right)=-\rho_{2}+\frac{q \rho_{3}}{2 k T}
$$

where $n_{a p}$ is apparent ideality factor (experimental data), and the coefficients $\rho_{2}$ and $\rho_{3}$ quantify the voltage deformation of the $\mathrm{BH}$ distribution, that is, the voltage dependencies of the mean $\mathrm{BH}$ and the barrier distribution widths are given by coefficients $\rho_{2}$ and $\rho_{3}$, respectively. The experimental $\Phi_{b o}$ versus $1 / 2 \mathrm{kT}$ and $n_{a p}$ versus $1 / 2 \mathrm{kT}$ plots are shown in Figure 9. The linearity in the apparent barrier height or ideality factor versus $1 / 2$ $\mathrm{kT}$ curves is in agreement with the recent model which is related to thermionic emission over a Gaussian distribution. The plot of $\Phi_{b o}$ versus $1 / 2 \mathrm{kT}$ is a straight line with the intercept on the ordinate determining the zero mean BH $\bar{\Phi}_{b 0} \quad(\mathrm{~T}=0 \mathrm{~K})$ and the slope gives the standard deviation $\left(\sigma_{0}\right)$. The corresponding values are $0.80 \mathrm{eV}$ and $114 \mathrm{mV}$ for $\bar{\Phi}_{b 0} \quad(\mathrm{~T}=0)$ and $\sigma_{0}$ respectively. Moreover, as can be seen in the Figure 2, the experimental results of $\Phi_{a p}$ fit very well with the theoretical Equation

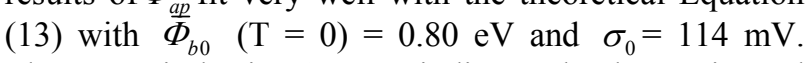
The open circles in Figure 2 indicates the data estimated with these parameters in using Equation (13) and filled circles indicates the experimental barrier heights measured from I-V characteristics. The observed standard deviation is $14.3 \%$ of the mean barrier height. The lower value of standard deviation shows the better rectifying performance with barrier homogeneity.

Figure 9 shows the experimental ideality factor versus $1 / 2 \mathrm{kT}$ plot is a straight line. The values obtained for $\rho_{2}$ and $\rho_{3}$ from the intercept and the slopes of the straight line are $0.2504 \mathrm{~V}$ and $-0.0141 \mathrm{~V}$, respectively. The linear behavior of this plot reveals that the ideality factor does indeed express the voltage deformation of the Gaussian distribution of the SBH. Furthermore, the experimental results of $n_{\text {ap }}$ fit very well with theoretical Equation (14) with $\rho_{2}=0.2504 \mathrm{~V}$ and $\rho_{3}=-0.0141 \mathrm{~V}$ as shown in Figure 3. The continuous solid line in the Figure 3 indicates data estimated with these parameters using Equation (14) and open circles indicates experimental ideality factor values. The lower value of $\sigma_{0}$ corresponds to more homogeneous barrier heights. According to Cavlet

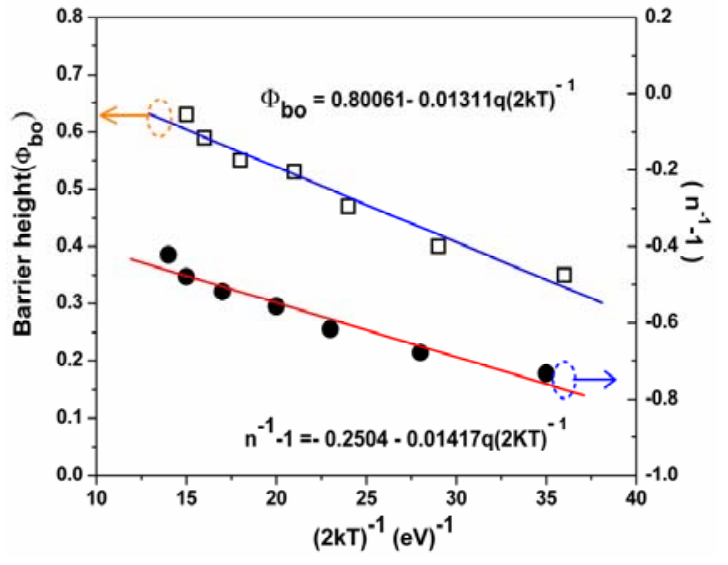

Figure 9. The zero-bias barrier height and ideality factor versus $1 / 2 \mathrm{kT}$ plots and their linear fits for the $\mathrm{Pd} / \mathrm{Ti} / \mathrm{n}-\mathrm{InP}$ SBD according to Gaussian distribution of the barrier heights.

et al. [43], the value of $\sigma_{0}(114 \mathrm{mV})$ is not small compared to the mean value of $\bar{\Phi}_{b 0}(\mathrm{~T}=0)=0.80 \mathrm{eV}$ which indicates the greater inhomogeneities at the interface and thus potential fluctuation. The inhomogeneity and the potential fluctuation only affect low temperature current-voltage characteristics.

Due to the barrier inhomogeneity at low temperatures, the conventional Richardson plot deviates from linearity. It can be modified by combining Equations (2) and (13) as follows

$$
\ln \left(\frac{I_{0}}{T^{2}}\right)-\left(\frac{q^{2} \sigma_{0}^{2}}{2 k^{2} T^{2}}\right)=\ln \left(A A^{*}\right)-\frac{q \bar{\Phi}_{b o}}{k T}
$$

A modified $\ln \left(\mathrm{I}_{0} / \mathrm{T}^{2}\right)-\left(q^{2} \sigma_{0}^{2} / 2 k^{2} T^{2}\right)$ versus $1000 / \mathrm{T}$ plot can be obtained according to Equation (15). The plot should give a straight line with the slope directly yielding the mean barrier height $\bar{\Phi}_{b 0} \quad(T=0)$ and the intercept (= $\ln \mathrm{AA}^{*}$ ) at the ordinate, determining $\mathrm{A}^{*}$ for a given diode area $A$. Figure 10 shows the modified $\ln \left(\mathrm{I}_{0} / \mathrm{T}^{2}\right)-$ $\left(q^{2} \sigma_{0}^{2} / 2 k^{2} T^{2}\right)$ versus $1000 / \mathrm{T}$ plot gives $\bar{\Phi}_{b 0} \quad(\mathrm{~T}=0)$, and $\mathrm{A}^{*}$ as $0.796 \mathrm{eV}$ and $6.16 \mathrm{~A} \cdot \mathrm{cm}^{-2} \cdot \mathrm{K}^{-2}$, respectively, without using the temperature coefficient of the barrier height $\alpha$. Mean while, this value of $\bar{\Phi}_{b 0}=0.796 \mathrm{eV}$ is approximately the same as the value of $\bar{\Phi}_{b 0}=0.80 \mathrm{eV}$ from the plot of $\Phi_{a p}$ versus $1 / 2 \mathrm{kT}$ given in Figure 9. The modified Richardson constant $\mathrm{A}^{*}=6.16 \mathrm{~A} \cdot \mathrm{cm}^{-2} \cdot \mathrm{K}^{-2}$ is in close agreement with the theoretical value of $\mathrm{A}^{*}=9.4$ $\mathrm{A} \cdot \mathrm{cm}^{-2} \cdot \mathrm{K}^{-2}$.

\subsection{Analysis of Capactance-Voltage- Temperature (C-V-T) Characteristics}

The experimental reverse bias $\mathrm{C}^{-2}-\mathrm{V}$ characteristics of the $\mathrm{Pd} / \mathrm{Ti} / \mathrm{n}$-InP SBD in the temperature range of 160 $400 \mathrm{~K}$ in steps of $40 \mathrm{~K}$ are shown in Figure 11. The 


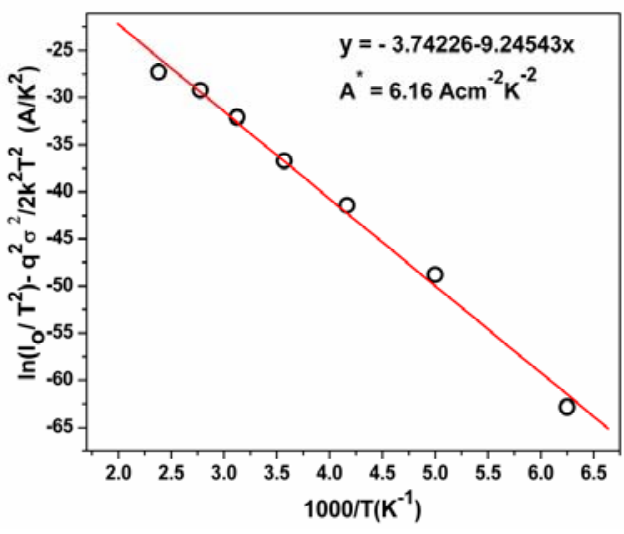

Figure 10. Modified Richardson $\ln \left(\mathbf{I}_{0} / \mathbf{T}^{2}\right)-\left(q^{2} \sigma_{0}^{2} / 2 k^{2} T^{2}\right)$ versus $1000 / T$ plot for the $P d / T i / n-I n P$ SBD according to the Gaussian distribution of barrier heights.

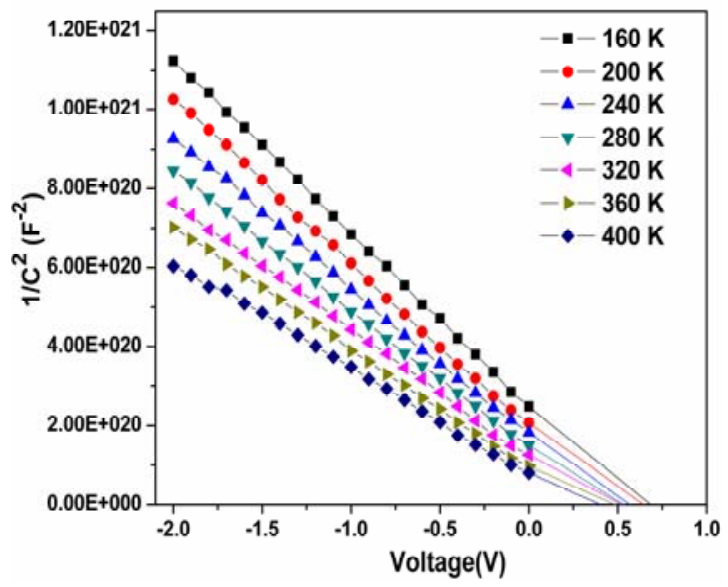

Figure 11. The reverse bias $C^{-2}-V$ characteristics of the $\mathrm{Pd} / \mathrm{Ti} / \mathrm{n}$-InP SBD in the temperature range of 160 - $400 \mathrm{~K}$.

junction capacitance has been performed at a frequency of $1 \mathrm{MHz}$. The $\mathrm{C}-\mathrm{V}$ relationship for Schottky diode is $[1,29]$

$$
\frac{1}{C^{2}}=\left(\frac{2}{\varepsilon_{s} q N_{d} A^{2}}\right)\left(V_{b i}-\frac{k T}{q}-V\right)
$$

where $\epsilon_{\mathrm{s}}$ is the permittivity of the semiconductor $\left(\epsilon_{\mathrm{s}}=\right.$ $12.4 \in_{0}$ ) [24], $V$ is the applied voltage. The $\mathrm{x}$-intercept of the plot of $\left(1 / \mathrm{C}^{2}\right)$ versus $V$ gives $V_{0}$ and it is related to the built in potential $V_{b i}$ by the equation $V_{b i}=V_{0}+k T / q$, where $T$ is the absolute temperature. The $B H$ is given by the equation $\Phi_{C V}=V_{0}+V_{n}+k T / q$, here $V n=(k T / q) l n$ $\left(N_{c} / N_{d}\right)$. The density of states in the conduction band edge is given by $N_{c}=2\left(2 \pi \mathrm{m}^{*} \mathrm{kT} / \mathrm{h}^{2}\right)^{3 / 2}$, where $\mathrm{m}^{*}=$ $0.078 \mathrm{~m}_{\mathrm{o}}$ and its value is $5.7 \times 10^{17} \mathrm{~cm}^{-3}$ for $\mathrm{InP}$ at room temperature [2]. The temperature dependence of the experimental carrier concentration $\left(N_{d}\right)$ is calculated from the slope of reverse bias $\mathrm{C}^{-2}-\mathrm{V}$ characteristics from Fig- ure 11 and the values of $N_{d}$ varied from $1.96 \times 10^{15}$ to $3.11 \times 10^{15} \mathrm{~cm}^{-3}$ in the temperature range of $160-400 \mathrm{~K}$. The values of $N c$ varied from $2.06 \times 10^{17}$ to $8.22 \times 10^{17}$ $\mathrm{cm}^{-3}$ as temperature varied between $160 \mathrm{~K}$ and $400 \mathrm{~K}$, respectively. It is observed that carrier concentration for $\mathrm{n}$-InP increased with increase in temperature. The estimated Schottky barrier height of Pd/Ti Schottky contact is in the range of $0.73 \mathrm{eV}$ at $160 \mathrm{~K}$ to $0.61 \mathrm{eV}$ at $400 \mathrm{~K}$ respectively. It is noted that the barrier height $\Phi_{C V}$ increased with decrease in temperature. Furthermore, as can be seen from Figure 2 it was observed that the $\Phi_{C V}$ values are higher than the $\Phi_{b o}$ values in the investigated temperature range. This discrepancy could be explained by the existence of an interfacial layer or of trap states in the semiconductor and the existence of Schottky barrier height inhomogeneity $[10,11,26]$. Due to the square dependence of $\Phi_{C V}$ on $1 / \mathrm{C}$, compared to the logarithmic dependence of $\Phi_{I-V}$ on the current, $\Phi_{C V}$ is more sensitive to the experimental error of the measurement data than $\Phi_{I V}$ [19]. Moreover, it is clearly seen from the Figure 2 that $\Phi_{C V}$ is obtained to increase with decreasing temperature. The temperature dependence of $\Phi_{C V}$ is expressed as

$$
\Phi_{C V}=\Phi_{C V}(T=0 \mathrm{~K})+\alpha T
$$

where $\Phi_{C V}(T=0 \mathrm{~K})$ is the barrier height extrapolated to zero temperature and $\alpha$ is the temperature coefficient of the barrier height. The linear fit of Equation (17) to the experimental data (filled squares) in Figure 2 yields $\Phi_{C V}(T=0 \mathrm{~K})=0.8114 \mathrm{eV}$ and $\alpha=-5.1 \times 10^{-4}$ $\mathrm{eVK}^{-1}$ which is the temperature coefficient of the InP band gap [44].

Furthermore, it can be seen that the apparent barrier height from the experimental forward bias I-V plot is also related to the mean barrier height $\bar{\Phi}_{b 0}=\bar{\Phi}_{C V}$ from the experimental reverse bias $\mathrm{C}^{-2}-\mathrm{V}$ plot $[10,40]$. The capacitance depends only on the mean band bending and is insensitive to the standard deviation $\sigma_{0}$ of the barrier distribution $[10,40]$. The relationship between $\Phi_{a p}$ and $\Phi_{C V}$ is given by $[10,40]$

$$
\Phi_{C V}-\Phi_{a p}=-\frac{q \sigma_{0}^{2}(T=0)}{2 k T}+\frac{q \alpha_{\sigma}}{2 k}
$$

where $\alpha_{\sigma}$ is attributed to the temperature dependence of $\sigma_{0}$ Figure 12 shows the experimental $\left(\Phi_{C V}-\Phi_{I V}\right)$ versus $1 / T$ plot according to Equation (18). The plot should give a straight line of the slope $\sigma_{0}^{2} / 2 k$ and a y-axis intercept $\alpha_{\sigma} / 2 k$ from which the parameters $\sigma_{0}$ and $\alpha_{\sigma}$ can be determined. The slope and y-axis intercept of the plot given the values of $\sigma_{0}=149 \mathrm{mV}$ and $\alpha_{\sigma}=5.59 \times$ $10^{-5} \mathrm{~V}^{2} \cdot \mathrm{K}^{-1}$, respectively. The value of $\sigma_{0}$ in the investigated temperature region is in close agreement with the value of $\sigma_{0}=114 \mathrm{mV}$ from the plot of $\Phi_{a p}$ versus $1 / 2$ 


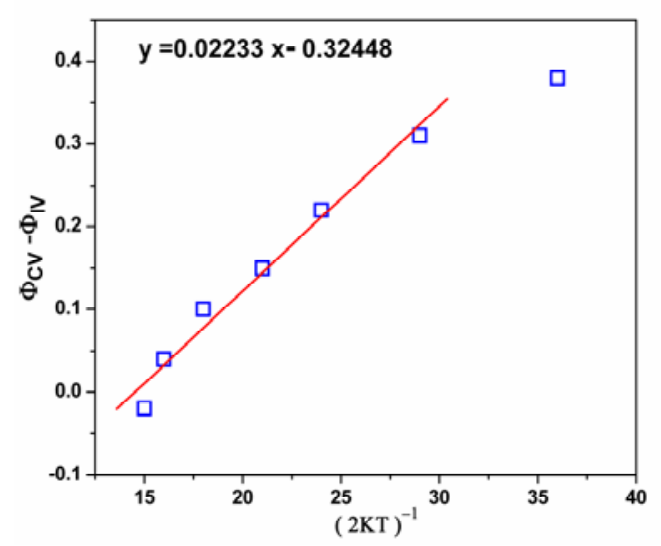

Figure 12. Barrier height difference between values as derived from the conventional evaluation of $I-V$ and $C-V$ data as a function of inverse temperature.

kT given in Figure 9 which is not small when compared to the mean $\mathrm{BH}$ value of $0.796 \mathrm{eV}$. Therefore, these significantly large potential fluctuations drastically affect low temperature I-V data and, in particular, they could be responsible for the curved behaviour of the conventional Richardson plot as shown in Figure 6.

\section{Conclusions}

In this paper, the current-voltage (I-V) and capacitance-voltage $(\mathrm{C}-\mathrm{V})$ characteristics of $\mathrm{Pd} / \mathrm{Ti} / \mathrm{n}$-InP SBDs have been investigated in the temperature range 160 $400 \mathrm{~K}$. The electrical parameters such as ideality factor (n) and zero-bias BH ( $\left.\Phi_{b o}\right)$ are found to be strongly temperature dependent. It is found that the ideality factor (n) of the diode decreases while the corresponding zero-bias SBH increasing with an increase in temperature. The values of series resistance $\left(R_{\mathrm{s}}\right)$ estimated from Cheung's method were strongly temperature dependent. The flat-band barrier height values are obtained from the temperature dependence of the I-V characteristics and the values are in the range $1.13-0.94 \mathrm{eV}$. The laterally homogeneous $\mathrm{SBH}$ value is approximately $0.71 \mathrm{eV}$ for the $\mathrm{Pd} / \mathrm{Ti} / \mathrm{n}-\mathrm{InP} \mathrm{SBD}$ which is deduced from the linear relationship between the experimental BHs and ideality factors. The mean $\mathrm{BH}\left(\bar{\Phi}_{b 0}\right)$ and effective Richardson constant $\mathrm{A}^{*}$ are found as $0.796 \mathrm{eV}$ and $6.16 \mathrm{Acm}^{-2} \mathrm{~K}^{-2}$, respectively, from a modified $\ln \left(I_{0} / T^{2}\right)-\left(q^{2} \sigma_{0}^{2} / 2 k^{2} T^{2}\right)$ versus $1000 / \mathrm{T}$ plot. The experimental results of $\Phi_{a p}$ and $n_{\text {ap }}$ fit very well with the theoretical equations related to the Gaussian distribution of $\Phi_{a p}$ and $n_{\text {ap. }}$. It can be concluded that the temperature dependent current-voltage $(\mathrm{I}-\mathrm{V})$ and capacitance-voltage $(\mathrm{C}-\mathrm{V})$ characteristic of the $\mathrm{Pd} / \mathrm{Ti} / \mathrm{n}$-InP Schottky barrier diodes over a wide temperature range have been explained on the basis of thermionic emission mechanism by assuming the pres- ence of Gaussian distribution of barrier heights.

\section{References}

[1] E. H. Rhoderick and R. H. Williams, "Metal-Semiconductor Contacts," 2nd Edition, Clarendon Press, Oxford, 1988.

[2] R. H. Williams and G. Y. Robinson, "Physics and Chemistry of III-V Compound Semiconductor Interfaces," C. W. Wilmsen, Ed., Plenum Press, New York, 1985.

[3] R. T. Tung, "Recent Advances in Schottky Barrier Concepts," Materials Science and Engineering: $R$, Vol. 35, No. 1-3, November 2001, pp. 1-138.

[4] K. Hattori and Y. Torii, "A New Method to Fabricate $\mathrm{Au} / n$-type InP Schottky Contacts with an Interfacial Layer," Solid-State Electronics, Vol. 34, No. 5, May 1991, pp. 527-531.

[5] Z. J. Horvath, V. Rakovics, B. Szentpali and S. Puspoki, "Schottky Junctions on n-Type InP for Zero Bias Microwave Detectors," Physica Status Solidi C, Vol. 3, February 2003, pp. 916-921.

[6] T. S. Huang and R. S. Fang, "Barrier Height Enhancement of Pt $/ n$-InP Schottky Diodes by $\mathrm{P}_{2} \mathrm{~S}_{5} /\left(\mathrm{NH}_{4}\right)_{2} \mathrm{~S}$ Solution Treatment of the InP Surface," Solid-State Electronics, Vol. 37, No. 8, August 1994, pp. 1461-1466.

[7] G. Eftekhari, "Electrical Characterization of Rapidly Annealed Ni and Pd/n-InP Schottky Diodes," Semiconductor Science and Technology, Vol. 10, No. 8, May 1995, p. 1163.

[8] M. Soylu, B. Abay and Y. Onganer, "The Effects of Annealing on Au/Pyronine-B/MD n-InP Schottky Structure," Journal of Physics and Chemistry of Solids, Vol. 71, No. 9, September 2010, pp. 1398-1403.

[9] Y. P. Song, R. L. Van Meirhaeghe, W. H. Laflere and F. Cardon, "On the Difference in Apparent Barrier Height as Obtained from Capacitance-Voltage and Current-VoltageTemperature Measurements on $\mathrm{Al} / p$-InP Schottky Barriers," Solid-State Electronics, Vol. 29, No. 6, June 1986, pp. 633-638.

[10] J. H. Werner and H. H. Guttler, "Barrier Inhomogeneities at Schottky Contacts," Journal of Applied Physics, Vol. 69, No. 3, February 1991, p. 1522.

[11] R. T. Tung, "Electron Transport at Metal-Semiconductor Interfaces: General Theory," Physical Review B, Vol. 45, No. 23, June 1992, p. 13509.

[12] A. Gumus, A. Turut and N. Yalcin, "Temperature Dependent Barrier Characteristics of CrNiCo Alloy Schottky Contacts on n-Type Molecular-Beam Epitaxy GaAs," Journal of Applied Physics, Vol. 91, No. 1, January 2002, p. 245.

[13] Y. G. Chen, M. Ogura and H. Okushi,“Temperature Dependence on Current-Voltage Characteristics of Nickel/ Diamond Schottky Diodes on High Quality Boron-Doped Homoepitaxial Diamond Film," Applied Physics Letters, Vol. 82, No. 24, June 2003, p. 4367.

[14] M. K. Hudait, P. Venkateswaralu and S. B. Krupanidhi, 
"Electrical Transport Characteristics of $\mathrm{Au} / \mathrm{n}-\mathrm{GaAs}$ Schottky Diodes on n-Ge at Low Temperatures," SolidState Electronics, Vol. 45, No. 1, January 2001, pp. 133141.

[15] F. A. Padovani, "Semiconductors and Semimetals," R. K. Willardson, A. C. Beer, Ed., Academic Press, New York, Vol. 7A, 1971.

[16] C. R. Crowell, "The Physical Significance of the $T_{0}$ Anomalies in Schottky Barriers," Solid-State Electronics, Vol. 20, No. 3, March 1977, pp. 171-175.

[17] R. T. Tung, J. P. Sullivan and F. Schrey, "On the Inhomogeneity of Schottky Barriers," Materials Science and Engineering: B, Vol. 14, No. 3, August 1992, pp. 266-280.

[18] R. F. Schmitsdorf, T. U. Kampen and W. Monch, "Explanation of the Linear Correlation between Barrier Heights and Ideality Factors of Real Metal-Semiconductor Contacts by Laterally Nonuniform Schottky Barriers," Journal of Vacuum Science \& Technology B, Vol. 15, No. 4, July-August 1997, p. 1221.

[19] S. Zhu, R. L. van Meirhaeghe, C. Detavernier, F. Cardon, G. P. Ru, X. P. Qu and B. Z. Li, "Barrier Height Inhomogeneities of Epitaxial $\mathrm{CoSi}_{2}$ Schottky Contacts on n-Si (100) and (111)," Solid-State Electronics, Vol. 44, No. 4, April 2000, pp. 663-671.

[20] H. Cetin and E. Ayyildiz, "Temperature Dependence of Electrical Parameters of the Au/n-InP Schottky Barrier Diodes," Semiconductor Science and Technology, Vol. 20, No. 6, May 2005, pp. 625-631.

[21] M. B. Reddy, A. A. Kumar, V. Janardhanam, V. Rajagopal Reddy and P. Narasimha Reddy, "Current-VoltageTemperature (I-V-T) Characteristics of $\mathrm{Pd} / \mathrm{Au}$ Schottky Contacts on n-InP (111)," Current Applied Physics, Vol. 9, No. 5, September 2009, pp. 972-977.

[22] M. Soylu and B. Abay, "Barrier Characteristics of Gold Schottky Contacts on Moderately Doped n-InP Based on Temperature Dependent $I-V$ and $C-V$ Measurements," Microelectronic Engineering, Vol. 86, No. 1, January 2009, pp. 88-95.

[23] A. Ashok Kumar, V. Janardhanam, V. R. Reddy and P. Narasimha Reddy, "Evaluation of Schottky Barrier Parameters of $\mathrm{Pd} / \mathrm{Pt}$ Schottky Contacts on n-InP (100) in Wide Temperature Range," Superlattices and Microstructures, Vol. 45, No. 1, January 2009, pp. 22-32.

[24] F. E. Cimilli, H. Efeoglu, M. Saglam and A. Turat, "Temperature-Dependent Current-Voltage and Capacitance-Voltage Characteristics of the Ag/n-InP/In Schottky Diodes," Journal of Material Science: Materials in Electronics, Vol. 20, February 2008, pp. 105-112.

[25] S. S. Naik and V. Rajagopal Reddy, "Analysis of Current-Voltage-Temperature $(I-V-T)$ and CapacitanceVoltage-Temperature $(C-V-T)$ Characteristics of $\mathrm{Ni} / \mathrm{Au}$ Schottky Contacts on n-Type InP," Superlattices and Microstructures, Vol. 48, No. 3, September 2010, pp. 330342.

[26] J. P. Sullivan, R. T. Tung, M. R. Pinto and W. R. Graham, "Electron Transport of Inhomogeneous Schottky Barriers: A Numerical Study," Journal of Applied Physics, Vol. 70, No. 12, December 1991, p. 7403.
[27] S. Zeyrek, S. Altindal, H. Yuzer and M. Bulbul, "Current Transport Mechanism in $\mathrm{Al} / \mathrm{Si}_{3} \mathrm{~N}_{4} / \mathrm{p}$-Si (MIS) Schottky Barrier Diodes at Low Temperatures," Applied Surface Science, Vol. 252, No. 8, February 2006, pp. 2999-3010.

[28] S. K. Cheung and N. W. Cheung, "Extraction of Schottky Diode Parameters from Forward Current-Voltage Characteristics," Applied Physics Letters, Vol. 49, No. 2, July 1986, p. 85.

[29] S. M. Sze, "Physics of Semiconductor Devices," 2nd Edition, John Wiley and Sons, New York, 1981.

[30] S. Chand and J. Kumar, "Current Transport in $\mathrm{Pd}_{2} \mathrm{Si}$ / n-Si(100) Schottky Barrier Diodes at Low Temperatures," Applied Physics A, Vol. 63, No. 2, March 1996, p. 171.

[31] N. Newman, M. V. Schilfgaarde, T. Kendelwicz, M. D. Williams and W. E. Spicer, "Electrical Study of Schottky Barriers on Atomically Clean GaAs(110) Surfaces," Physical Review B, Vol. 33, No. 2, January 1986, p. 1146.

[32] Z. J. Horvath, "Comment on "Analysis of $I-V$ Measurements on $\mathrm{CrSi}_{2}$ - Si Schottky Structures in a Wide Temperature Range," Solid-State Electronics, Vol. 39, No. 1, January 1996, pp. 176-178.

[33] F. Pandovani and R. Stratton, "Field and ThermionicField Emission in Schottky Barriers," Solid-State Electronics, Vol. 9, No. 7, July 1966, pp. 695-707.

[34] C. R. Crowell and V. L. Rideout, "Normalized Thermionic-Field (T-F) Emission in Metal-Semiconductor (Schottky) Barriers," Solid-State Electronics, Vol. 12, No. 2, February 1969, pp. 89-105.

[35] Z. J. Horvath, V. Rakovics, B. Szentpali, S. Puspoki and K. Zdansky, "InP Schottky Junctions for Zero Bias Detector Diodes," Vacuum, Vol. 71, No. 1-2, May 2003, pp. 113-116.

[36] J. Osvald, "New Aspects of the Temperature Dependence of the Current in Inhomogeneous Schottky Diodes," Semiconductor Science and Technology, Vol. 18, No. 4, April 2003, p. L24.

[37] F. E. Jones, B. P. Wood, J. A. Myers, C. H. Daniels and M. C. Lonergan, "Current Transport and the Role of Barrier Inhomogeneities at the High Barrier n-InP Poly (Pyrrole) Interface," Journal of Applied Physics, Vol. 86, No. 11, December 1999, p. 6431.

[38] M. K. Hudait, P. V. Venkateswaralu and S. B. Krupanidhi, "Electrical Transport Characteristics of $\mathrm{Au} / \mathrm{n}$ GaAs Schottky Diodes on n-Ge at Low Temperatures," Solid-State Electronics, Vol. 45, No. 1, January 2001, pp. 133-141.

[39] J. H. Werner and H. H. Guttler, "Temperature Dependence of Schottky Barrier Heights on Silicon," Journal of Applied Physics, Vol. 73, No. 3, February 1993, p. 1315.

[40] H. H. Guttler and J. H. Werner, "Influence of Barrier Inhomogeneities on Noise at Schottky Contacts," Applied Physics Letters, Vol. 56, No. 12, March 1990, p. 1113.

[41] S. Zhu, R. L. Van Meirhaeghe, S. Forment, G. P. Ru, X. P. Qu and B. Z. Li, "Schottky Barrier Characteristics of Ternary Silicide $\mathrm{Co}_{1-x} \mathrm{Ni}_{x} \mathrm{Si}_{2}$ on $\mathrm{n}$-Si(100) Contacts 
Formed by Solid Phase Reaction of Multilayer," SolidState Electronics, Vol. 48, No. 7, July 2004, pp. 12051209.

[42] R. F. Schmitsdorf, T. U. Kampen and W. Monch "Correlation between Barrier Height and Interface Structure of Ag/Si(111) Schottky Diodes," Surface Science, Vol. 324, No. 2-3, February 1995, pp. 249-256.

[43] L. E. Calvet, R. G. Wheeler and M. A. Reed, "Electron
Transport Measurements of Schottky Barrier Inhomogeneities," Applied Physics Letters, Vol. 80, No. 10, March 2001, p. 1761.

[44] Y. F. Tsay, B. Gong and S. S. Mitra, "Temperature Dependence of Energy Gaps of Some III-V Semiconductors," Physical Review B, Vol. 6, No. 6, September 1972, p. 2330. 